\title{
outlook
}

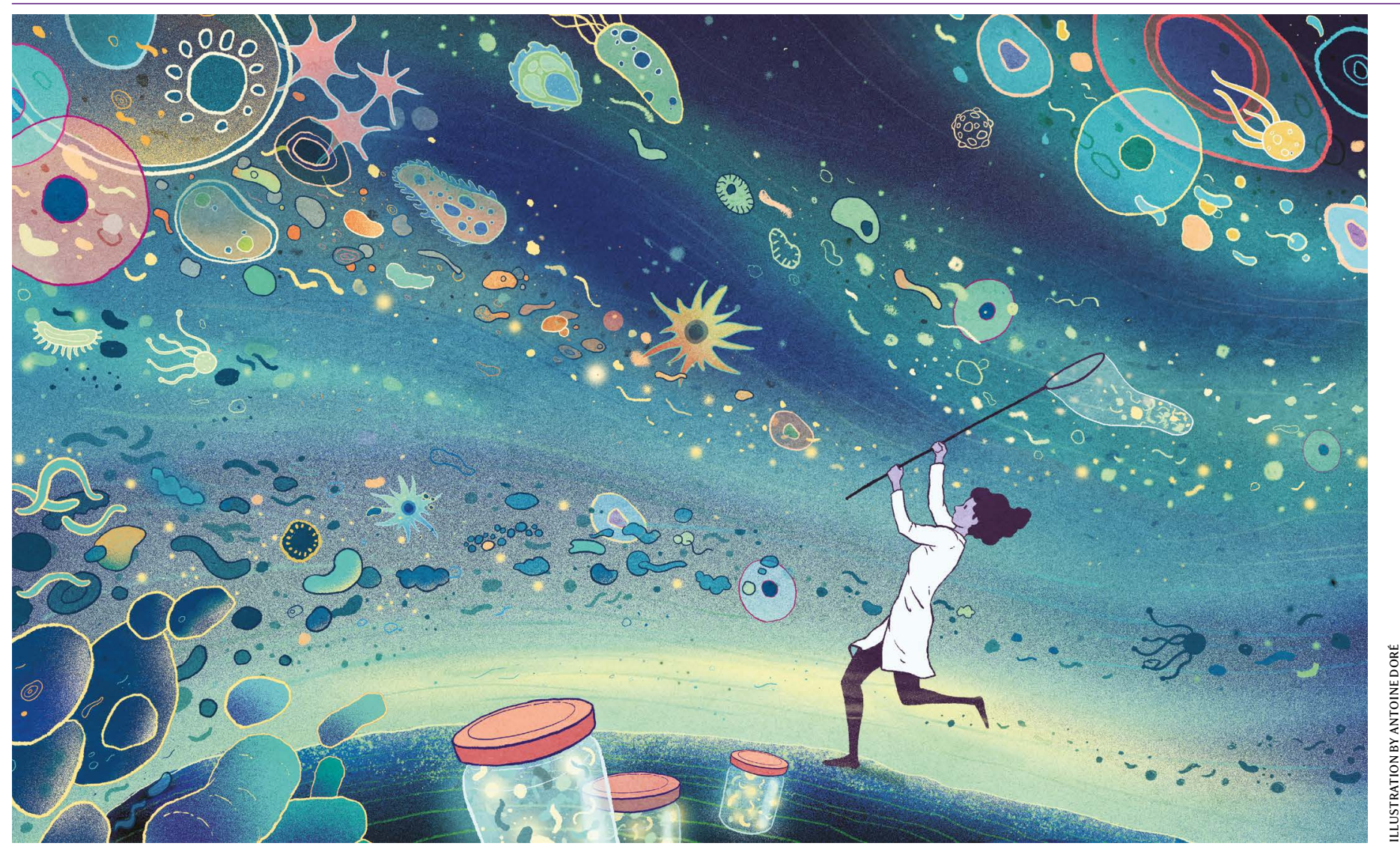

\section{The hunt for a healthy microbiome}

\section{Despite evidence of the gut microbiome's role in human health, researchers are still working out what shapes the community of microbes. By Michael Eisenstein}

W hat does a healthy forest look like? A seemingly thriving, verdant wilderness can conceal signs of pollution, disease or invasive species. Only an ecologist can spot problems that could jeopardize the longterm well-being of the entire ecosystem.

Microbiome researchers grapple with the same problem. Disruptions to the community of microbes living in the human gut can contribute to the risk and severity of a host of medical conditions. Accordingly, many scientists have become accomplished bacterial naturalists, labouring to catalogue the startling diversity of these commensal communities. Some $500-1,000$ bacterial species reside in each person's intestinal tract, alongside an undetermined number of viruses, fungi and other microbes.

Rapid advances in DNA sequencing technology have accelerated the identification of these bacteria, allowing researchers to create 'field guides' to the species in the human gut. "We're starting to get a feeling of who the players are," says Jeroen Raes, a bioinformatician at VIB, a life-sciences institute in Ghent, Belgium. "But there is still considerable 'dark matter'."

Currently, these field guides are of limited use in distinguishing a healthy microbiome from an unhealthy one. Part of the problem is the potentially vast differences between the microbiomes of apparently healthy people. These differences arise through a complex combination of environmental, genetic and lifestyle factors. This means that relatively subtle differences can have a disproportionate role in determining whether an individual is relatively healthy or at increased risk of developing disorders such as diabetes. Understanding the clinical implications of those differences is also a challenge, given the extensive interactions between these microbes, and with their host, as well as the conditions in which that individual lives. "One person's healthy microbiome might not be healthy in another context - it's a tricky concept," says Ruth Ley, a microbial ecologist at the Max Planck Institute for Developmental Biology in Tübingen, Germany.

Researchers such as Ley are trying to better understand the forces that shape the human gut microbiome - both in the modern era, and across evolutionary history. The emerging picture indicates that even if there is no one healthy microbiome, there are ample opportunities for our lifestyle to interfere with the proper function of these complex commensal communities. And to understand how the breakdown of these ecosystems drives disease, researchers will need to move beyond microbial field guides and begin dissecting how these species interact with their hosts and with each other. 
A mother's first gift to her newborn is a healthy smattering of microbes. Some are passed along through breastfeeding and skin-to-skin contact, but many microbes are acquired during passage through the birth canal. This means that if the baby is delivered by caesarean section, they might miss out on a valuable bacterial starter kit. Because a child's earliest years generally establish the composition of a gut community that will persist throughout adulthood, the resulting disruptions can have serious long-term health consequences. "As these infants grow, they have higher risk of obesity, and of modern plagues like diabetes, allergies and asthma," says Maria Gloria Dominguez-Bello, a microbiologist at Rutgers University in New Brunswick, New Jersey. In a small clinical study, her team found that swabbing newborns delivered by c-section with fluids from their mother's birth canal could help to mitigate some of the lost microbial diversity ${ }^{1}$. Several larger trials are under way to assess the longer-term health benefits.

Environmental exposures early in life also strongly affect a child's microbiome. Susan Lynch, a microbiome researcher at the University of California, San Francisco, has been exploring links between environmental factors during childhood and the subsequent risk of developing allergies and asthma. Her findings indicate that new parents shouldn't be afraid of a little dirt - or fur. After monitoring a cohort of nearly 1,200 infants, Lynch and her colleagues found that a dog might be a baby's best friend when it comes to avoiding respiratory disorders ${ }^{2}$. "The only factor that discriminated high-from low-risk groups was dog ownership," says Lynch. She says that dogs (and, to a lesser extent, cats) "increase the diversity of bacteria and lower the diversity of fungi in the houses where these babies are raised". This finding aligns with other research showing that a rural upbringing or growing up on a farm might yield a richer gut microbiome that reduces the risk of inflammatory respiratory diseases relative to children raised in more urban environments.

At a certain point during childhood, the composition of the gut microbiome generally stops changing - although precisely when is unclear. A study in 2012 surveyed gut microbes from individuals in Malawi, Venezuela and the United States, and found a striking pattern ${ }^{3}$. "By three, you can no longer tell the babies from the adults," says Dominguez-Bello, who was a co-author on the paper. However, she notes that there is also evidence that the microbiome remains somewhat mutable beyond this point. What is clear is that by adulthood, this ecosystem reaches a state of equilibrium. “It's very stable," says Eran Segal, a computational biologist at the Weizmann Institute of Science in Rehovot, Israel. “We see changes, but you will still look mostly similar, even over many years."

Some of the changes seen in adulthood are driven by environment and lifestyle. In a 2018 study of 1,046 ethnically diverse adults living in Israel, Segal demonstrated microbial differences that had little to do with ethnicity ${ }^{4}$. "Environmental inputs could account for $20-25 \%$ of the variability in the microbiome," says Segal. Drugs are an obvious source of disruption, and antibiotics - taken either deliberately to fight infection or unwittingly in processed foods - can profoundly affect the microbiota. Even drugs with no clear role in controlling bacteria can cause perturbations. Raes notes that one major European microbiome study was confounded by unexpected effects from the diabetes drug metformin ${ }^{5}$.

Diet is also a powerful external influence, even if the precise mechanisms by which it exerts its effects remain unclear. One study in 2018 found that immigrants to the United States from Thailand experienced a striking 'westernization' of their gut flora - a transformation that could be, at least in part, attributed to adopting a US diet ${ }^{6}$.

\section{Mismatched to modernity}

The changes observed in immigrants from Thailand were accompanied by increased risk of obesity. The study did not establish a causal link, but the results are consistent with an increasingly popular hypothesis that urbanization - and modern life in general - might be highly disruptive to the tight-knit relationship that has evolved between humans and their microbes. "We have made the assumption that the Western microbiome of a healthy person is a healthy microbiome," says microbiologist Justin Sonnenburg at Stanford University in California. Instead, he and others think that the intersection of diet, antimicrobial precautions and general hygiene leads to a culling of the gut community, and that this disruption might contribute to the elevated risk of chronic disease in industrialized societies. "This combination of Western diet and depleted microbiome has likely led to a simmering inflammatory state," Sonnenburg says.

Several studies have identified a stark difference between the microbiota of urban populations and those of Indigenous populations that lead traditional agrarian or hunter-gatherer lifestyles, which more closely resemble those of our early ancestors. These differences seem to be attributable mainly to loss of bacterial diversity, which might be linked to the lack of fibre in Western diets. The Hadza, a population of hunter-gatherers living in Tanzania, eat 100-150 grams of dietary fibre per day, Sonnenburg says - ten times as much as a typical person in the United States. As a result, fibre-digesting bacteria such as those belonging to the genus Prevotella, which can form up to $60 \%$ of the gut microbiome in non-Western populations, are much less abundant in the United States. Sonnenburg's team has demonstrated how these changes can become firmly entrenched in a population over the course of just a few generations ${ }^{7}$. Mice colonized with human microbiota and fed a low-fibre diet lost microbial species that remained in mice eating a high-fibre diet. When the offspring of the low-fibre-diet mice were given a high-fibre diet, the species loss was reversible, but after four generations, the missing bacteria were gone for good.

Katherine Amato, an anthropologist at Northwestern University in Evanston, Illinois, has been trying to get to the evolutionary root of a healthy human microbiome by studying non-human primates and tracing the effects of changes in human lifestyle and physiology. In general, Amato says, similarities in microbiome composition among primate species are closely mapped to their evolutionary relatedness. But in a 2019 comparative analysis, Amato found that components of the human microbiota (in particular, microbes from people living in non-industrialized societies) did not map as closely as expected to those of our nearest relatives - the great apes, chimpanzees and bonobos ${ }^{8}$. Instead, the microbiota bore a striking resemblance to those of baboons - a more distant relation,

\section{“One person's healthy microbiome might not be healthy in another context."}

but one that has a lifestyle more similar to that of early humans. "Most great apes are living in rainforests and eating fruit diets," says Amato, "but we tend to think of our ancestors as living in open woodlands or savannah habitats, and eating an omnivorous diet - like baboons". This suggests that dietary and environmental factors have played a prominent part in shaping the human microbiome.

Ley thinks that the microbiome offers a powerful mechanism for adapting quickly to lifestyle changes - at least, relative to the normal glacial pace of evolution. Indeed, her group has found evidence of microbiome adaptation in response to the evolution of lactose tolerance ${ }^{9}$ and digestion of highstarch diets - genetic adaptations that have emerged only in certain populations over the 


\section{outlook}

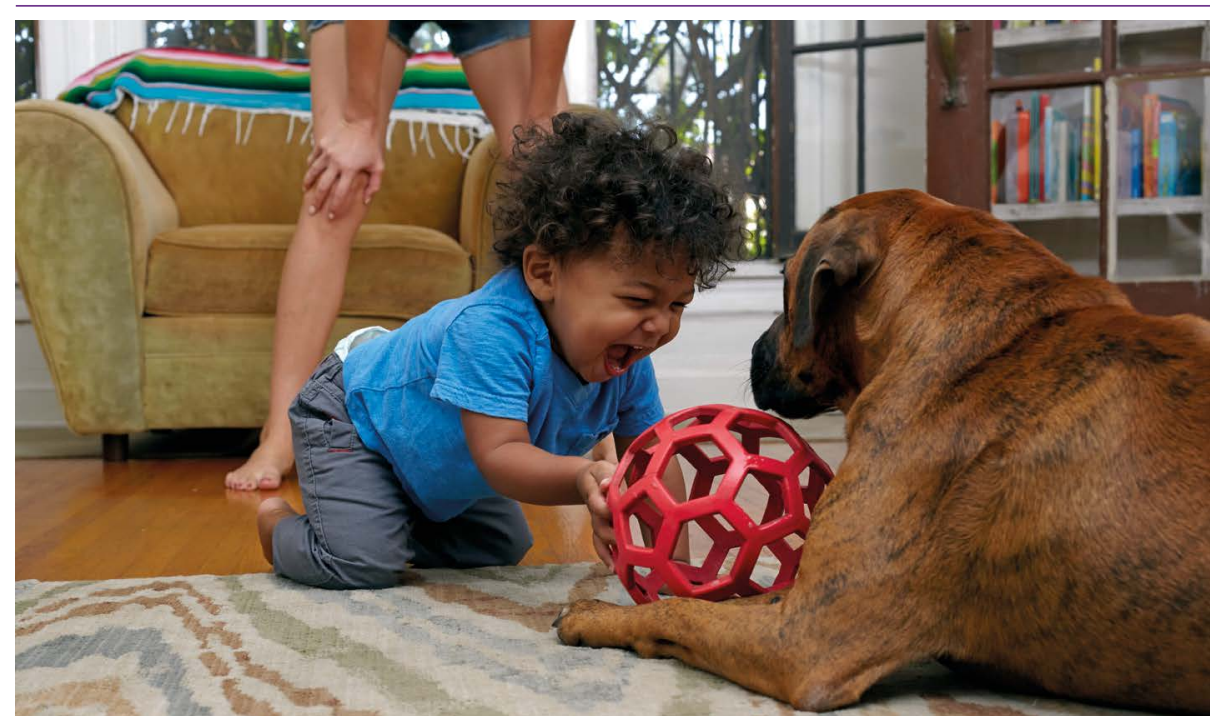

Growing up with a dog in the house increases the diversity of bacteria children are exposed to.

past 10,000 years or so. But if changes happen quickly, as demonstrated by the rapid industrialization that occurred over the past few centuries, the historically healthy relationship between host and microbiome could become maladaptive as species that the body might have evolved to rely on are lost. "Antibiotics and sanitation have been key in controlling infectious diseases," says Dominguez-Bello, "but have the collateral, unintended consequences of harming our good microbes."

\section{Seeing the forest}

Although researchers have gained a better understanding of what human gut microbiomes look like, they are still struggling to pin down which components are essential to our well-being. One problem is that there are far too few data sets to allow researchers to draw statistically robust connections between the microbiome and health or disease. Segal draws a comparison with the human genome - only when many high-quality sequences were available did it begin to offer clinical value. "There are probably 30 million people that have been genome sequenced up until today, while in the microbiome there are around 10,000 samples publicly available," he says.

This issue is compounded by the geographic bias in microbiome data. Beyond a handful of studies of selected groups such as the Hadza, most data are from the United States, Europe and China. "We know very little about microbiome variation in Africa, southeast Asia and South America," says Raes. That information gap will be especially relevant for understanding the extent of the suggested 'missing microbes' problem in the industrialized world.

A larger, more global data set would give a better-informed starting point for broadly a healthy individual can look like - and thus make it easier to recognize disease-linked perturbations. But researchers also need to move beyond studies that simply assess correlation on the basis of presence or absence of a specific microbe in a healthy individual or a person with a disease at a particular point in time.

There are now a number of multi-year, longitudinal studies that monitor both the health and the microbiome composition of many individuals over extended periods. The Canadian Healthy Infant Longitudinal Development study, for example, is monitoring more than 3,400 children over the course of 5 years in an effort to identify factors that contribute to conditions such as asthma and allergies. "If we can see that a microbiome change precedes a clinical change, then maybe we can establish causality," says Segal. Such patterns would give clinicians more confidence in the potential value of a diagnostic result or intervention, and would be invaluable for studying the contribution of the microbiome to chronic conditions that manifest gradually, such as diabetes.

Researchers are also making their bacterial censuses more detailed. Early microbiome investigations were limited by the narrow range of intestinal species that scientists could grow in the lab. But the plummeting cost of sequencing has made it possible to capture detailed snapshots of the DNA extracted from faecal microbes. Researchers can now go beyond species level to identify strains of bacteria, and even genomic variants in those strains. Sonnenburg, for example, is using this approach to look for mutations that might affect the metabolic activity or dietary preferences of different gut microbes. understanding what a normal microbiome in
Many microbes still slip through the net, however. Standard methods of microbiome analysis favour identification of bacteria, and are not as good at identifying other common gut microorganisms. "We rarely see signatures of fungi in our data, but we know they're there," says Lynch. "And we know that they're contributing to the overall interaction between microbiome and host." Alternative microbiome-analysis techniques offer a workaround. Harvesting and analysing RNA rather than DNA, for example, allows investigators to capture changes in gene expression that can reveal dysfunction in ostensibly normal gut species. "A perfectly nice-looking microbiome might be doing things that aren't healthy," says Ley. Other researchers are turning to metabolomic techniques - comprehensive chemical analysis of the various biomolecules produced in a microbiome sample. This is allowing researchers to eavesdrop on how microbes are communicating with each other and with their host's cells. "These molecules are the end products," says Lynch. "That's where the meat is in trying to define biomarkers of a healthy microbiome." Her lab has made important strides with such approaches, including homing in on a microbial lipid known as 12,13-diHOME, which seems to be a driver of inflammation in infants at high risk of asthma ${ }^{10}$.

Such data might offer the best readout yet of how well our internal ecosystem is thriving - essentially, inspecting the soil, water and leaves of the forest, rather than simply counting the trees. "There won't be 'the' healthy microbiome, just like there's no perfect genome," says Segal. "There could be multiple healthy configurations." These profiles of microbial activity might prove the fastest route to validating hypotheses on microbiome function and dysfunction, and accelerate the translation of discoveries into clinical trials. "The time of observation hasn't come to an end, but I think it's really time to move to interventions," says Raes. "You can only understand a system if you give it a good kick and see what happens."

Michael Eisenstein is a science journalist in Philadelphia, Pennsylvania.

\footnotetext{
1. Dominguez-Bello, M. G. et al. Nature Med. 22, 250-253 (2016).

2. Havstad, S. et al. J. Allergy Clin. Immunol. 128, 880-885 (2011).

3. Yatsunenko, T. et al. Nature 486, 222-227 (2012)

4. Rothschild, D. et al. Nature 555, 210-215 (2018).

5. Forslund, K. et al. Nature 528, 262-266 (2015).

6. Vangay, P. et al. Cell 175, 962-972 (2018).

7. Sonnenburg, E. D. et al. Nature 529, 212-215 (2016).

8. Amato, K. R. et al. Genome Biol. 20, 201 (2019).

9. Goodrich, J. K. et al. Cell Host Microbe 19, 731-743 (2016)

10. Levan, S. R. et al. Nature Microbiol. 4, 1851-1861 (2019).
} 